\title{
Adaptive Haptically Informed Assembly with Mobile Robots in Unstructured Environments
}

\author{
P. Devadass ${ }^{\mathrm{a}}$, S. Stumm ${ }^{\mathrm{a}}$ and S. Brell-Cokcan ${ }^{\mathrm{a}}$ \\ ${ }^{a}$ Chair for Individualized Production, RWTH Aachen University Germany \\ E-mail: office@ip.rwth-aachen.de
}

\begin{abstract}
-
Robot assisted construction processes in the architectural domain which include assembly are uncommon due the size difference of the robot with respect the scale of the output. In order to extend the workspace of industrial robots, these can be mounted on top of a mobile platform. However industrial mobile robotics currently focuses on the utilization within clearly defined and structured production environments. Nevertheless, due to increasing product variety, a paradigm shift away from repetition of static task towards dynamic human robot collaboration is noticeable. Especially mobile robots face very specific challenges such as inaccuracy, dynamic on-site adaptability and predictability of whether the design is producible within the constraints of the robot. In this paper we discuss these challenges encountered due to onsite construction through a built project and illustrate the solution taken forward to address these challenges. In this research we propose a new methodology for onsite construction of non-standard components using mobile robots. The demonstrated project comprises of complex space frame timber system where every component of the structure is unique in its shape and size. For this we combine pre-planning of design with human-robot collaboration for on-site adaptation. The approach utilizes force torque sensors embedded within the robot in combination with haptic fiducials, in order to improving accuracy of the robotic fabrication and allow for human-robot collaboration within assembly. Employing the a-priori design knowledge the robot places the work-piece at the correct angle, while allowing for human adaptation of the path in order to increase accuracy. The paper illustrates the various optimization techniques developed to predict design manufacturability including potentially necessary adaptions. The research envisions a safe and automated large-scale construction methodology for complex systems and opens to new gateways for construction, allowing the collaboration between human workers and mobile robots within unstructured environments.
\end{abstract}

Keywords -

Mobile Robotics; Onsite Construction; Haptic Feedback; Re-informed design; Human-Robot Collaboration; Haptic Programming

\section{Introduction}

Low level automation is becoming common practice for pre-fabrication of building elements in construction industries. Simultaneously concepts from Industry 4.0 allow for more and more custom production specifically in fabrication of individualized elements. However, construction industry still struggles to develop effective techniques for onsite construction processes especially for assembly due to the limited working envelope of a stationary robot as well as limited adaptability in robotics in order to handle raw material with high tolerances. But with the advent of mobile robotics, the possibility of onsite construction seems promising. However, there are several factors which limit the use of these tools.

One critical factor is the design being developed independently of fabrication process constraints of these complex machines. Hence the designer does not realize the limitations of the fabrication setup while developing complex designs. This results in fabrication difficulties or even non-manufacturable designs. In a commercial practice, the designer typically sends the design directly to a fabricator, who tries to find quick solutions and intuitively fabricates by experience which results in a one-off solution for each design. But in case of mass production of individualized components an automated workflow is necessary. In the project developed by Aarhus School of Architecture, Israel Institute of Technology and the ETH Zurich [1], which has proved the value of integrating topology optimization for direct realization in the digital process. The research pavilion, 2011 of ICD Stuttgart further demonstrates the use of structural optimization for the full-scale fabrication of plated timber structures using bespoke robotic milling. Current robotic fabrication methods carried out at ETH Zurich under the Robotic Timber Construction group [2], as well as the Digital Urban Orchid by IAAC [3] achieve 
similar output. However, these projects do not address the direct influence of fabrication process and its parameters towards the design, as well as the potential of a reciprocal relationship between fabrication and design. Similarly, the Wood Chip Barn project by Hooke Park [4] although harvesting the inherent structural form of the timber fail to automate the challenges of using a limited size robot for oversized workpieces (tree forks). At the time of writing, there are no existing design methods in timber construction where the machine and fabrication workspace parameters and its constraints are integrated into the digital design process which informs the design.

To overcome the gap between design and fabrication, we introduce a novel concept of design which is driven by a continuous production feedback and the integration of fabrication constraints. The fabrication constraints are addressed within the domain of robot's reachability for the given design. This methodology is demonstrated through 'The Twisted Arch' project (Figure.1). The potentials and limitations of each fabrication resource are analyzed through design and construction of a 1:1 scale prototype of complex space frame timber structures by employing a mobile robotic setup and a bandsaw cutting process for fabrication, as well as human robot collaborated assembly. The outcome of the research results in the creation of an intelligent computational program, which provides visual guidance for the user during the design process. The project was first introduced in [4].

Another critical factor to be considered while using mobile robots is the inaccuracies encountered due to the movement of the mobile platform which could be caused due to uneven floor, dirt, etc. This requires an additional element which compensates the accumulated errors of all elements in the structure. In our research, a construction feedback method is implemented where the inaccuracies of fabrication of each workpiece is automatically measured using haptic sensors and the errors are then compensated in the subsequent assembly of the structure. Thus, avoiding errors accumulation within the process establishing a continuous design and process feedback loop.

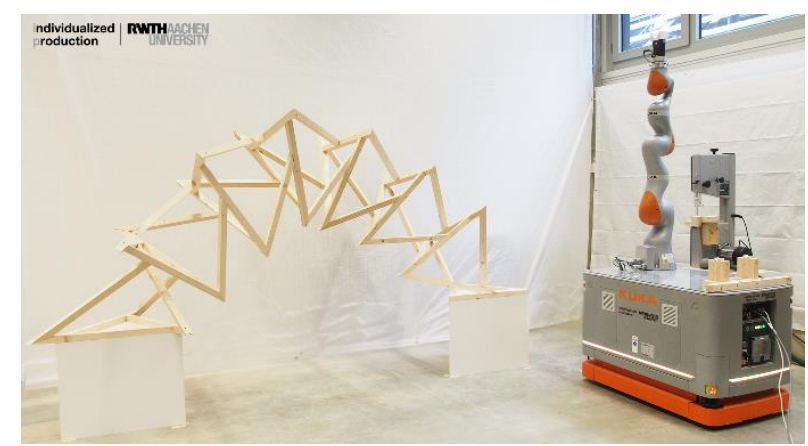

Figure 1. 'Twisted Arch' Project

\section{Concept and Workflow}

The general workflow and concept of our approach, as illustrated within Figure 2 based on our demonstrator project, consists of the following elements:

- $\quad$ Global Design (the overall structure to be fabricated)

- Assembly workspace analysis based on the global design ensuring reachability and collision free assembly, as well as structural stability

- Extraction of a single work pieces informed by the global design

- Fabrication workspace analysis to ensure manufacturability with feedback into the global design

- Execution of design to fabrication workflow with dynamic calibration resulting in feedback to the workpiece design

- Execution of design to assembly workflow with human-assisted adaption resulting in feedback to the global design

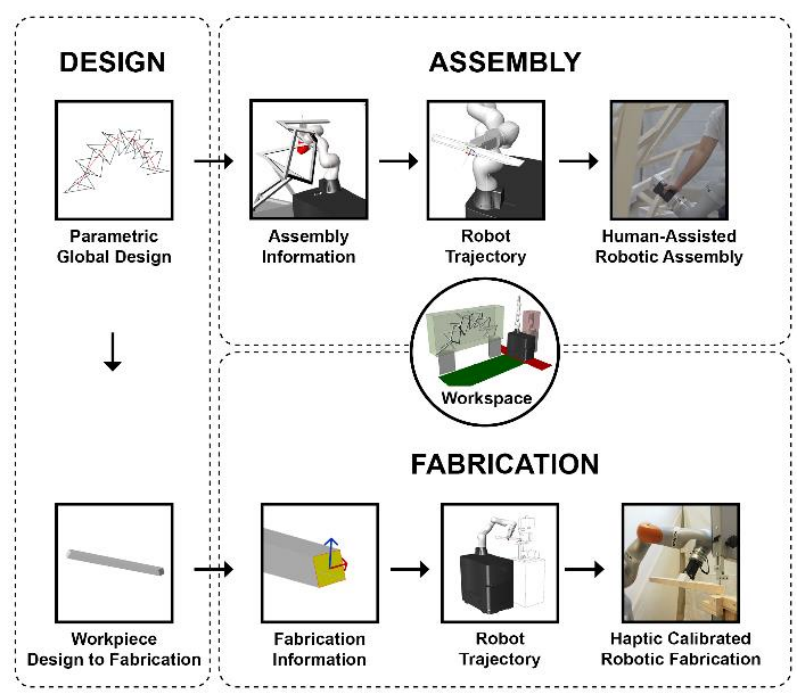

Figure 2. Process Overview

Within the following this workflow will be discussed in detail based on 'The Twisted Arch' project. Therefore, a first proof of concept is introduced, which illustrates the potential of bidirectional feedback between design, fabrication and assembly both in the context of workpiece fabrication as well as global structural design. Within [6] a first approach to transfer workspace constraints towards human-robot collaboration within assembly was introduced, specifically through the integration of constraint models within the robot controller and the term haptic programming was coined. The architectural impact of this workflow, with specific focus on the continuous design and process feedback loop, will be further discussed in section 10 . 


\section{Global Design}

The design of the prototype is based on a simple catenary arch comprising of a complex triangular space frame system made of timber elements. To test the process' influence on design, a complex timber space frame design connected by simple butt joints which follows a user-defined curve, is developed parametrically in a CAD environment. The parameters which include span, height, and offset of the catenary arch are optimized to test the workspace of the mobile robotic assembly process within the limits of structural stability of the design. The optimization in turn radically changes the space frame design and subsequently changes the length and angle of the cut of each element while still maintaining the configuration of the system. The maximum reach of the robot is verified to generate the working envelope and subsequently the robot's workspace. This data is continuously fed to the designer through custom visualization to make an informed choice for an ideal design. The entire interface is developed in the Rhinoceros3D and Grasshopper3D environment. The algorithms are custom plugins to check the functionality of the process developed by the authors. The design is initially simplified using the centerlines of the components and system for easier computation.

Another algorithm informs the design towards structural stability with the help of Karamba, a thirdparty plugin in grasshopper developed by Clemens Preisinger. The plugin integrates timber properties, in this case beech as part of the structural simulation. Irregularities like rot, cracks, etc. are not considered in process. The displacement of potential designs is then visualized to allow the designer to make an informed choice for the ideal design. The space frame is generated along the Cantenary arch allowing the design to choose different space frame variations, which are in turn used to generate solid geometries from the structural centerlines. Both algorithms simultaneously, inform the user to evaluate the design in terms of fabricability and structurally (Figure.3).

The design is evaluated every time to identify the non-manufacturable components through another iterative algorithm. The algorithm uses inverse kinematic solver, namely KUKA|prc [8], which checks for reachability, collisions, and singularities of the robot simulation. Nevertheless, after a series of optimization procedures, there are instances where the robot is still not able to manufacture the components (for example if the workpiece exceeds the fabrication limitations such as workpiece length, the angle of cut or a combination of both). The algorithm then highlights the geometries which cannot be manufactured in red. This visual illustration informs the designer whether the design can be fabricated or not, throughout the design process. The design can be continuously altered by the designer through quick modifications of the curve until all components are manufacturable. The designs generated are recorded and analyzed to understand deviation of the manufacturable design from the one intended by the designer.

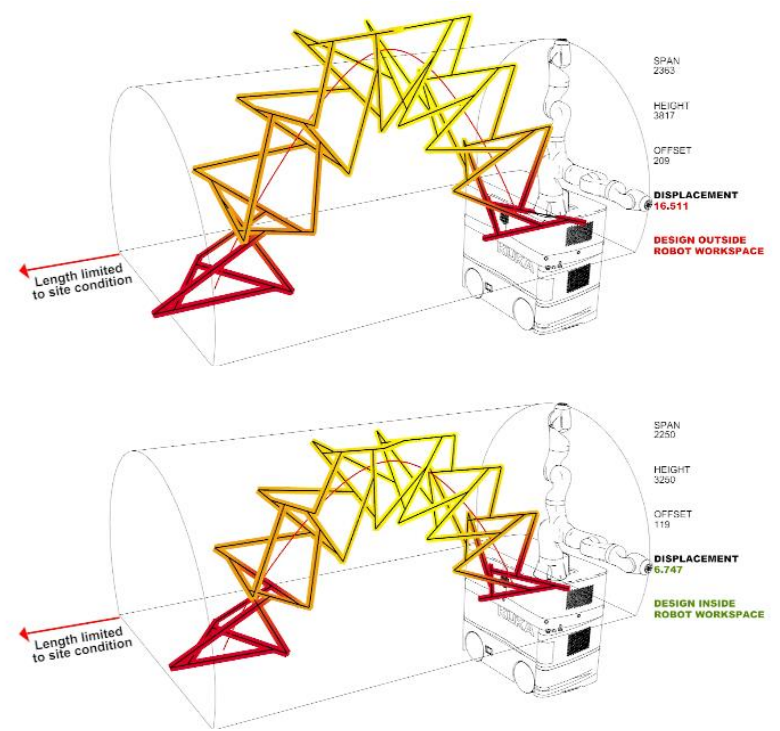

Figure 3. Optimization of the Design guided by the robotic workspace and structural constraints

This process establishes a new methodology in the field of architecture where every design decision is continuously informed and controlled by the parameters of fabrication. The process results in multiple options where every design is completely manufacturable but with slight modifications in its overall appearances from the one initially envisioned by the designer. The biggest drawback of this method is that the algorithm does not indicate which exact parameters are to be modified and by how much to achieve a manufacturable design. Although the constraints are identified and parameterized in this research, the next step is to develop an interface which would result in providing the above-mentioned feedback to the designer. The next steps are also to develop an algorithm to find the best suitable robot position, tool position and orientation of end-effector. This would result in an alternative approach where an optimal machine setup is generated based on the design created which otherwise would be a local solution for a given setup.

\section{Workpiece: Design to Fabrication}

After several iterations an optimized design is finalized, and an algorithm creates simple butt joinery between connecting components from the centerlines of the space frame system. Butt joinery is generally used to 
connect two more timber pieces when the ends are cut flat at specific angles where they meet. The flat faces of the butt joints are fastened using timber screws. The butt joinery results in compound angles at certain connections due to complexity of the design. The sectional dimensions of the timber stock are $35 \times 35 \mathrm{~mm}$. To avoid loss of the strength considering the limited sectional size of the timber, each timber component is restricted to only one connection at each end. The output from the algorithm is the final geometry which needs to be fabricated from the given stock material.

The length of each component is limited to a certain size depending on the maximum reach of the robotic arm or the robots working envelope. This is ensured to avoid collision between the timber material and the robotic arm or the environment. As the timber is gripped by the robot only at the center, the lengths of the timber pieces are limited to avoid extensive vibrations are the end of the stock material.

As the entire workflow is based on the centerline information and the local design (joinery) is automatically developed from it, this method allows transfer of the global design (timber framing drawing) made by another designer into the fabrication process. The centerlines of the connecting members in a design are required for the cad data.

\section{Demonstrator Process Overview}

Within the Twisted Arch project, the fabrication and assembly process flow consists of the following steps:

- $\quad$ Gripping of a square timber pieces from a supply station are manual handover.

- Optimization of the mobile robot's position towards the bandsaw with regard to reachability

- Haptic measurement adaption of the bandsaw plane to reduce the positioning error of the mobile platform

- Reorientation of the timber piece towards the bandsaw blade

- Cutting of the timber piece according to the compound angle

- Reorientation of the timber piece towards the band sawblade with the second side

- Cutting of the second side of the timber piece according to its compound angle

- Moving the piece away from the saw workstation

- Movement of the mobile platform towards the assembly station

- Movement of the cut timber piece towards the assembly position

- Manual adaption of the timber piece position to reduce inaccuracies

- Robot-assisted assembly
- Movement of the arm away from the assembly

- Movement of the mobile platform towards the bandsaw work station

- The assembly process is continued by retrieving the next timber piece.

\section{Workspaces}

The setup consists of a KUKA iiwa with $14 \mathrm{~kg}$ payload and maximum reach of $840 \mathrm{~mm}$ which has a gripper as an end effector, the robot is mount KUKA Mobile Robotic (KMR) platform, a standard workshop bandsaw with a maximum cutting depth of $110 \mathrm{~mm}$. Due to the limited reachability of the robot two boxes which provide the base on either ends of the arch at a raised height of $500 \mathrm{~mm}$ is constructed.

The major challenge of using stationary robot is developing a successful robot path devoid any collisions. In this process the challenge is amplified due to continuous repositioning of the mobile robot base. For mobile robots a dynamic workspace is considered, unlike in stationary robots with a static workspace. The actual workspace is dependent on the setup and therefore requires the superposition of various workspaces, such as robot, fabrication, assembly workspace and its surrounddings taking into consideration within ever-changing and unstructured construction environments.

Before we begin to understand the fabrication and assembly processes, one needs to consider the setup and various workspaces involved in production (Figure.4). To comprehend and implement the parameter space within which fabrication and assembly can be successfully carried out, the effective workspace of each production resource (machine, tool, material) is analyzed These analytical results are combined to create a process model that considers the interrelationship between all parts of the fabrication process. The fabrication workspace is a combination of robot, end effector (gripper) and tool workspace (bandsaw).

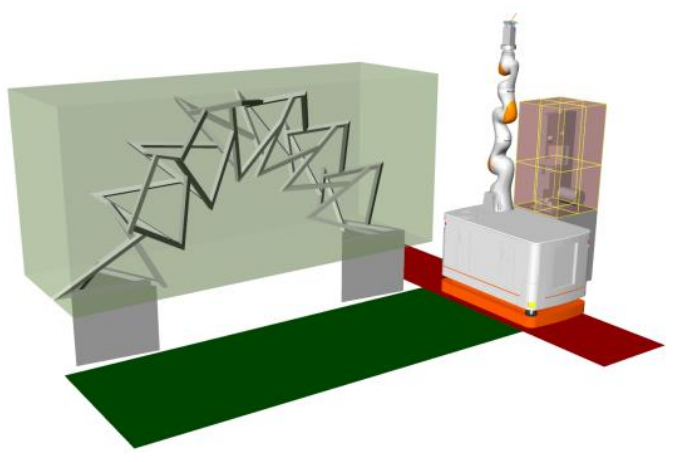




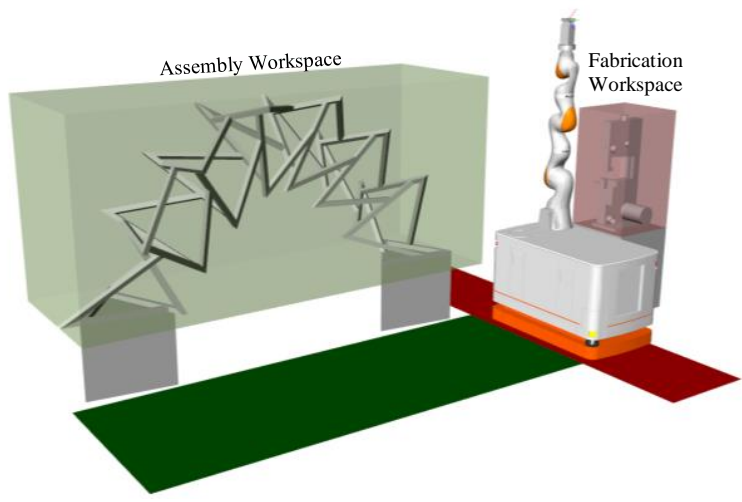

Figure 4. Workspaces

The robot workspace is the functional workspace which differs from working envelope of robot because the workspace takes the robots TCP (Tool Center Point) into account [7]. To determine the robot workspace an iterative algorithm is developed with the help of a genetic solver (namely Galapagos a Grasshopper3D plugin), which in turn generates some material and additional design constraints, such as timber section length and possible cutting angles. With the minimum and maximum reach known we can determine the threedimensional robot workspace.

The bandsaw workspace can be described as a collection of robots TCP positions for cutting. The intersection of the robot with end-effector and bandsaw workspace defines the fabrication workspace. By establishing this fabrication workspace, it is easier to test the manufacturability of a component. Comparative studies are conducted between various robot and tool positions to understand their impact on the workspace.

The intersection of robot with end-effector with various TCP positions for assembly of all components in the prototype define the assembly workspace. The sequential assembly order of the timber components must be considered and understood so that the robot workspace does not collide with assembly workspace of the surrounding components. Each process is clearly defined by the boundaries of fabrication and assembly workspaces which are placed orthogonally to each other so that the mobile platform is allowed to freely operate to resolve reachability issues without any collisions.

\section{Robot trajectory optimization for fabrication process}

While manually cutting timber using a bandsaw, tremendous amount of forces is exerted by the bandsaw blade on the workpiece. Therefore, the workpiece is ensured to rest on the bandsaw table so that the forces are mostly absorbed by the table. This allows the fabricator to easily guide the workpiece at the required direction and angle without experiencing much of the forces. Similarly, while using the robot, in order to ensure least amount of forces are transferred to the robotic arm, the workpiece is required to rest on the bandsaw table during the cut (Figure.5). This prerequisite becomes a major robot trajectory challenge as the robot not only has to place the workpiece at the required orientation and position but also ensure the workpieces rests on the bandsaw table.

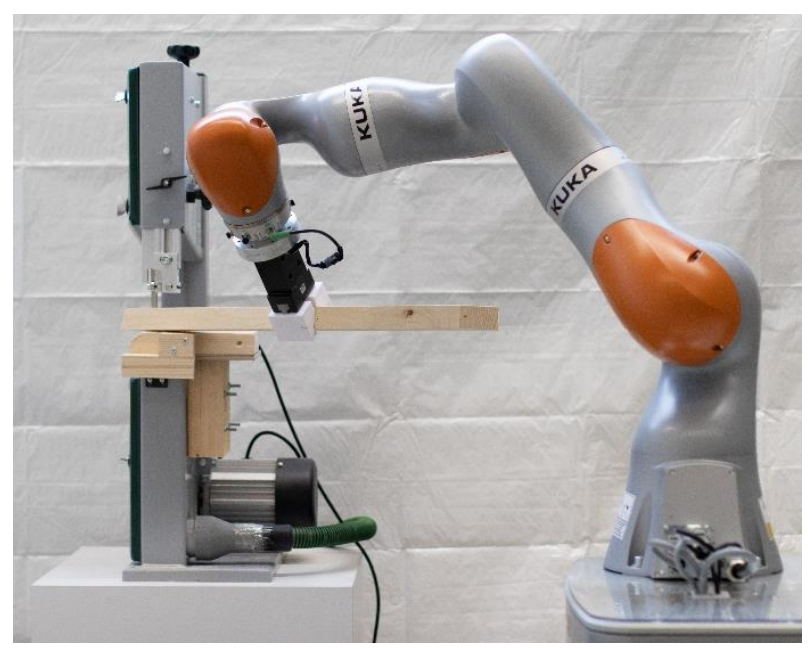

Figure 5. Resting the workpiece on the bandsaw table using the robot during fabrication

Hence, to achieve robot reachability for the above complex trajectory devoid of any collisions, a series of optimizations are executed. While using a stationary robot, a suitable gripping position from any of the four available directions of the workpiece is used to ensure reachability. Unfortunately, due to gripping and regripping of the workpiece every time for each cut of the workpiece, severe inaccuracies are encountered. To rest the workpiece on the bandsaw table, the workpiece geometry is rotated around the cutting surface until at least one edge (in case of compound angle cut) or a flat surface (in case of a simple angle cut) aligns with the table. However, in the current process to resolve this problem, we move the KUKA mobile platform, which in turn changes the base position of the KUKA iiwa and therefore enables different axis configuration in the robotic arm movement (Figure.6). This automatic optimization is conducted until a suitable trajectory is obtained which is free from collisions and well within the reach of the robot. 


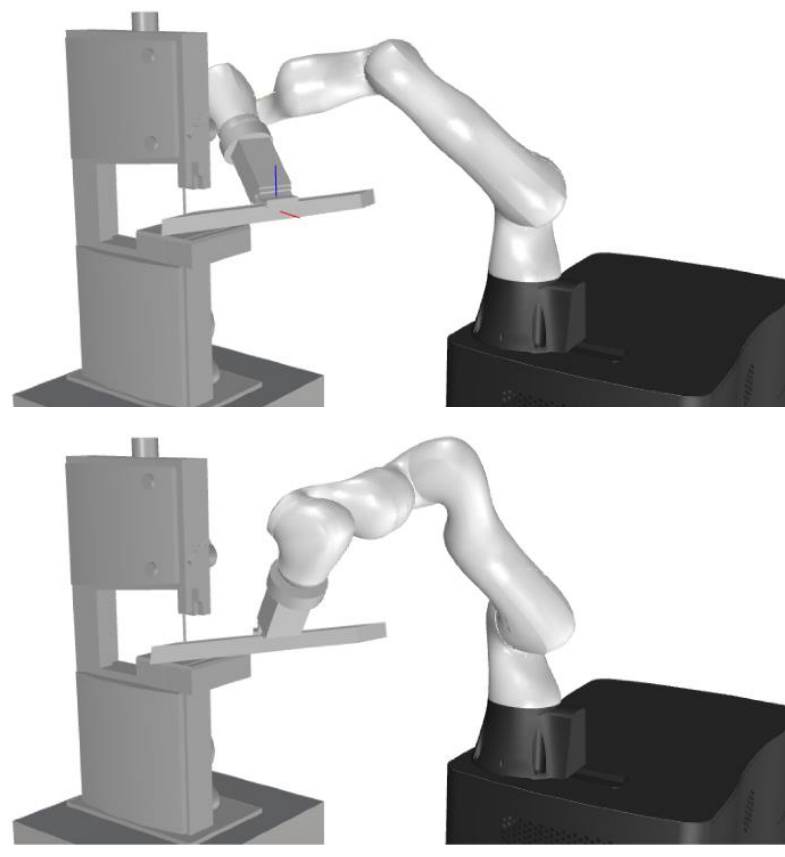

Figure 6. Optimizing the workpiece position to the robot and machine workspace

While the movement of the robotic arm is highly accurate the movement of the mobile platform is not. While it is possible to create a full map of the environment using the mobile platforms laser scanners this is however a very static process that does not allow for a dynamically changing environment as is often the case within construction. We compensate the inaccuracies within the process through haptic feedback for dynamic adaptivity on top of the statically planned fabrication process from design. In order to create dynamic adaptivity within the fabrication process, the robot is moved to a pre-calibrated position employing a haptic fiducial, before the cut is processed (Figure.7). Using the force torque sensors inbuilt in the robot, the robot dynamically calibrates the deviation between the haptic fiducial and the pre-calibrated position. By calculating this deviation, the cutting position is automatically updated to a corrected position in accordance with the real-world placement of the bandsaw thus providing an accurate cut. The pre-calibrated plane on the haptic fiducial serves as the primary reference point for the entire fabrication and assembly setup. All robot movements are referenced to it.

Without the haptic feedback for accuracy, the fabrication process observed an error over serval centimeters. The inaccuracies was inconsistent to evaluate as the mobile robot moved to various position depending the angle and size of the cut. But on an average the inaccuracies was found to be $20-30 \mathrm{~mm}$. With the haptic feedback the accuracy improved with an error less than $2.5 \mathrm{~mm}$.

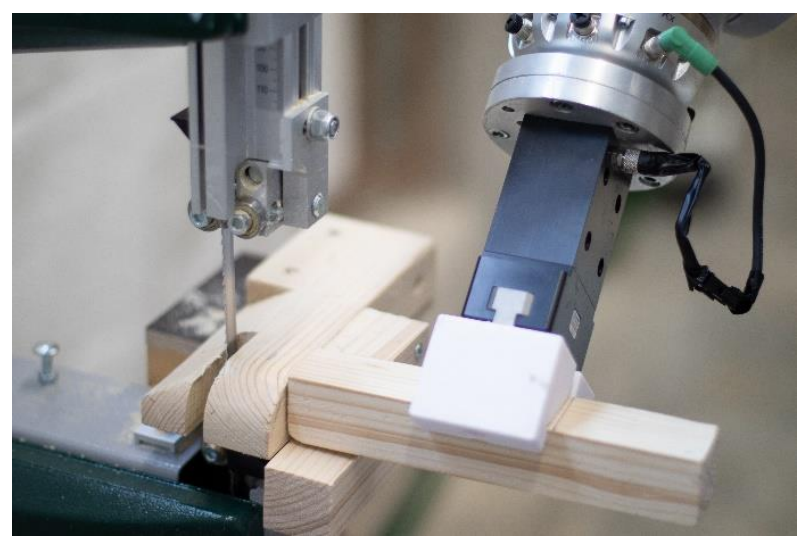

Figure 7. Haptic Feedback using the custom built fiducial

\section{Robot trajectory optimization for assembly process}

Current workflows consider design informed fabrication the construction of complex assembly however requires more consideration for sequence planning as well as adaptation towards on-site deviations. Within assembly deviations between pre-planned design and real-world construction increase with every layer. Similar to fabrication, to resolve reachability issues during assembly the mobile platform is moved closer to the position of the component (Figure.8), however while creating the structure there are no available haptic fiducials on or closer to the prototype to increase the mobile platforms accuracy.

There are instances where the robot is still unable to reach the target position. Since the geometry plane is placed along the centreline of the geometry, we can rotate it into four different configurations which leads the robot gripping the part from 4 different directions. This also changes the robot's configuration while keeping the geometry in the same place. In order to compensate for these deviations, the robot connects the assembly component to the existing butt joint of its predecessor. 


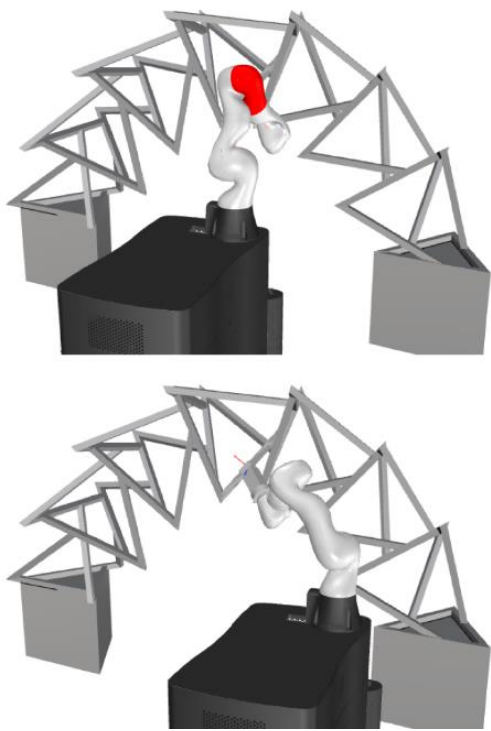

Figure 8. Optimizing the mobile robot position for reachability

However, the final assembly is conducted through human robot collaboration where the robot orients the workpiece in position while the human co-workers adapts the timber piece position in order to connect it correctly to the already fabricated space frame system (Figure.9). During assembly the robot is therefore set to an adaptive compliance mode, meaning the robot is in soft mode that allows the human co-worker to move the workpiece to a certain degree. If the deviations between planned target position and real-world position become too high the robot increases its stiffness and cannot be moved further of track. The components should be assembled in order so that they do not interfere with the assembly of its nearby components.

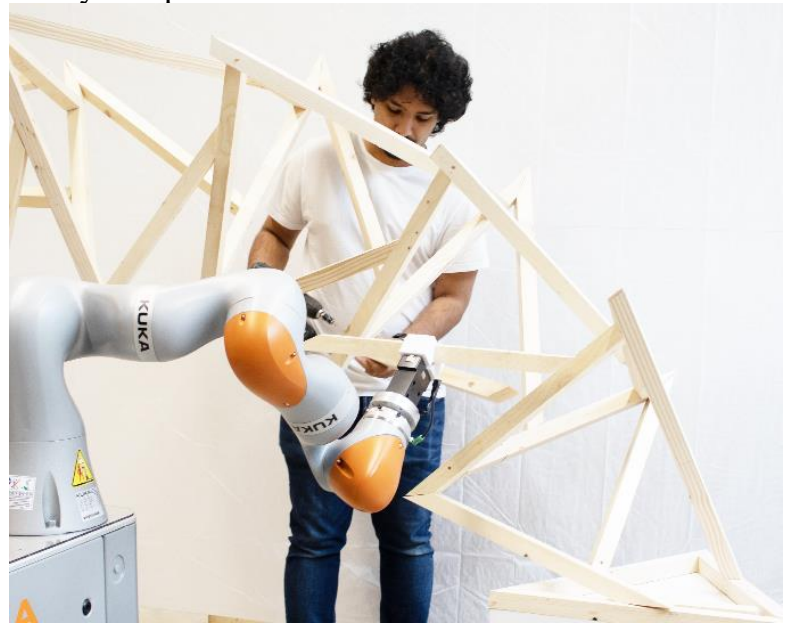

Figure 9. Human Robot Collaboration

\section{The IDAA Framework}

This leads to a reciprocal communication strategy between human co-worker and robot. This movement is easily guided by a user with its respective joinery to compensate the inaccuracies encountered during fabrication and transition, while simultaneously allowing the identification of construction errors that would lead to high deviations between design and assembly. The Production Immanent Design concepts of KUKA|prc which allow for a direct motion planning based on parametric design are therefore redefined and extended within the IDAA System, which was first introduced 2016 in [9] and further detailed in [5] for a stationary KUKA iiwa robot. However, within this paper the reciprocal relationship between the mobile platform and robotic arm movement is detailed.

In general, this system allows the integration haptic interaction primitives and concepts for dynamic configuration and path adaptation on-site, which is especially valuable for mobile robotics. The IDAA system does not rely on computer vision, which is unreliable in environments with dynamic lighting conditions but creates new means of communication between design and assembly through haptic feedback, not only for fully automated processes but also for human-robot collaboration.

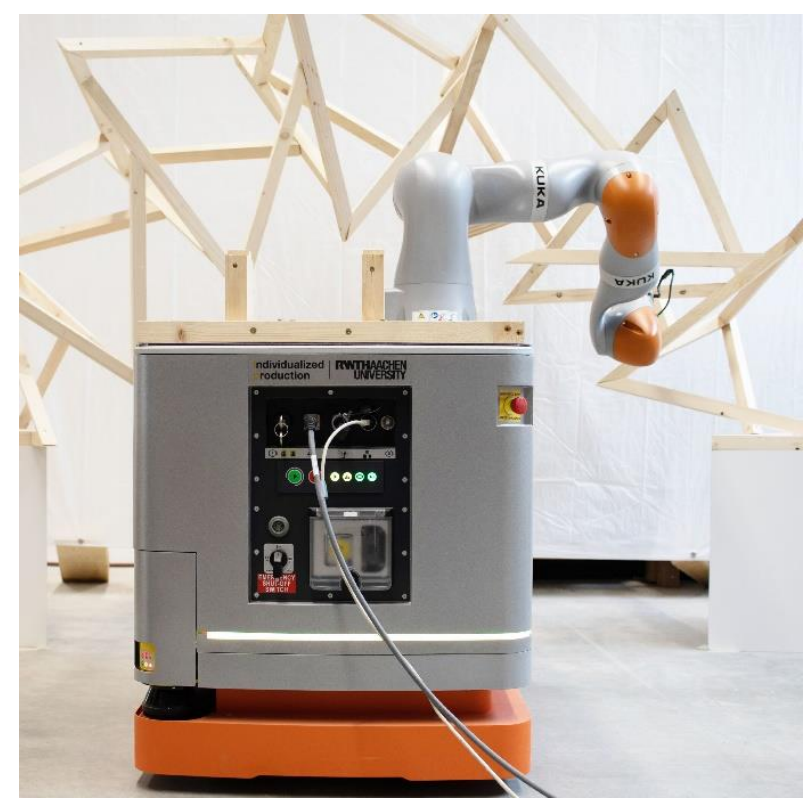

Figure 10. Robotic Assembly

The design of adaption and control strategies can be challenging especial for users without expertise in robotics. In order to allow for the use of haptic feedback for adaptation in fabrication as well as assembly, or even extending these control strategies with other sensory input, a superposition between the geometrically planned 
path and control strategy needs to be created.

Similar to skilled based robot programming [10] a catalogue of operations is created allowing the designer to link between geometric positioning and the execution of complex operations, such as dynamic recalibration of the robotic base frame at a haptic fiducial within fabrication. Such operations need to be accessible to designers but also create reusable descriptions of each operation. The IDAA Framework therefore allows for the extension of the operation catalogue in a combined approach the data interface to each operation is given by the geometric but localized path description, based on this the fitting control strategy can be chosen in a clearly defined setting.

\section{Architectural Impact}

The research illustrates how a single mobile robot can be resourcefully used for complex onsite construction processes and for multiple applications (including fabrication and assembly) and through efficient material handling in the 'Twisted Arch' project. Thus, demonstrating in what manner designers can thoughtfully develop design considering the parameters of the construction thereby yielding maximum efficiently from minimal resources. The research social impacts tangibly, by realizing the downsides of current technology and shows how human senses can be used sensibly for collaborative processes. Within the larger context of construction, the workflow establishes a new methodology in architectural design where the designer is always informed not only during design process but also during the construction process. One such example is the machinic inaccuracies encountered which is addressed through continuous designing and redesigning during the construction process. Thus, this methodology also allows the designer to have complete control throughout the process.

\section{Synopsis}

Within this work we illustrated the use of an adaptable mobile robot for onsite construction. By establishing a continuous design and process feedback loop the integration of a wide variety of fabrication and assembly tasks becomes, as well as individualized production becomes feasible. In order to make this approach applicable to other construction task further development is however necessary.

Within future work we will therefore focus on the automatic modelling of superimposed workspaces. By allowing for the representation and transfer of the necessary parameter space both from a production point of view as well as intended design adaptation can be optimized to adhere to the existing constraints from both viewpoints. While feedback from onsite production is possible this is still not fully integrated.

Furthermore, the used system is not suited for the rough environments of the construction site. The transfer to different systems and processes is still a major challenge and requires further research and development.

\section{References}

[1] Søndergaard, A.; Amir, O.; Eversmann, P.; Piskorec, L.; Stan, F.; Gramazio, F. and Kohler, M. 2016, "Topology Optimization and Robotic Fabrication of Advanced Timber Space-Frame Structures" Robotic Fabrication in Architecture,Art and Design, pp 190-203

[2] Willmann, J.; Knauss, M.; Bonwetsch, T.; Aleksandra Apolinarska, A.; Gramazio, F. and Kohler, M. 2015, "Robotic timber construction Expanding additive fabrication to new dimensions." Automation in Construction. 61.

[3] Figliola, A. et.al. 2016. "Digital Urban Orchard: robotic manufacturing of complex shape." Advances in Architectural Geometry 2016

[4] Mollica, Z. and Self, M., 2016, 'Tree Fork Truss: Geometric Strategies for Exploiting Inherent Material Form' in Adriaenssens, S., Gramazio, F., Kohler, M., Menges, A. and Pauly, M. (eds.), Advances in Architectural Geometry 2016

[5] Stumm, S.; Devadass, P. and and Brell-Cokcan, S., 2018 'Haptic programming in construction' in the Construction Robotics Journal Volume 2, pp. 3-13

[6] Stumm, S. and Brell-Cokcan, S., 2018 'Haptic programming' in Robotic Fabrication in Architecture, Art and Design 2018, pp 44-58

[7] Aggarwal, Luv, 2016 "Reconfigurable Validation Model for Identifying Kinematic Singularities and Reach Conditions for Articulated Robots and Machine Tools" (2014). Electronic Theses and Dissertations. Geometry 2016, Zurich, p.138-153

[8] Braumann, J., and Brell-Cokcan, S., 2011 "Parametric Robot Control: Integrated CAD/CAM for Architectural Design." In Integration through Computation: Proceedings of the 31st Annual Conference of the Association for Computer Aided Design in Architecture (ACADIA), pp. 242-251.

[9] Stumm S, Braumann J, Brell-Cokcan Sigrid (2016) Human-machine interaction for intuitive programming of assembly tasks in construction. In: 6th CIRP conference on assembly technologies and systems (CATS) procedia CIRP 44, pp 269-274

[10] M. R. Pedersen, L. Nalpantidis, R. S. Andersen, C. Schou, S. Bøgh, V. Krüger, and O. Madsen. Robot skills for manufacturing: From concept to industrial deployment. Robotics and Computer-Integrated Manufacturing, 37:282 - 291, 2016. 